\title{
Effect of the retinal size of a peripheral cue on attentional orienting in two- and three-dimensional worlds
}

\author{
Yizhou Jiang ${ }^{1} \cdot{\operatorname{Sijie~} \mathrm{Li}^{1} \cdot \text { You } \mathrm{Li}^{1} \cdot \operatorname{Hang} \text { Zeng }}{ }^{1} \cdot \mathrm{Qi}$ Chen ${ }^{1,2,3}$
}

Published online: 23 March 2016

(C) The Psychonomic Society, Inc. 2016

\begin{abstract}
It has been documented that due to limited attentional resources, the size of the attentional focus is inversely correlated with processing efficiency. Moreover, by adopting a variety of two-dimensional size illusions induced by pictorial depth cues (e.g., the Ponzo illusion), previous studies have revealed that the perceived, rather than the retinal, size of an object determines its detection. It remains unclear, however, whether and how the retinal versus perceived size of a cue influences the process of attentional orienting to subsequent targets, and whether the corresponding influencing processes differ between two-dimensional (2-D) and three-dimensional (3-D) space. In the present study, we incorporated the dot probe paradigm with either a 2-D Ponzo illusion, induced by pictorial depth cues, or a virtual 3-D world in which the Ponzo illusion turned into visual reality. By varying the retinal size of the cue while keeping its perceived size constant (Exp. 1), we found that a cue with smaller retinal size significantly facilitated attentional orienting as compared to a cue with larger retinal size, and that the effects were comparable between 2$\mathrm{D}$ and 3-D displays. Furthermore, when the pictorial background was removed and the cue display was positioned in either the farther or the closer depth plane (Exp. 2), or when both the depth and the background were removed (Exp. 3), the
\end{abstract}

Qi Chen

qi.chen27@gmail.com

1 Center for Studies of Psychological Application and School of Psychology, South China Normal University, Guangzhou, China

2 Guangdong Key Laboratory of Mental Health and Cognitive Science, South China Normal University, Guangzhou, China

3 Epilepsy Center, Guangdong 999 Brain Hospital, Guangzhou, China retinal size, rather than the depth, of the cue still affected attentional orienting. Taken together, our results suggest that the retinal size of a cue plays the crucial role in the visuospatial orienting of attention in both 2-D and 3-D.

Keywords Attentional orienting · Ponzo illusion · Retinal size $\cdot$ Perceived size $\cdot$ Three-dimensional (3-D)

The zoom lens model of attention proposes that the size of the attentional focus can be adjusted on the basis of the current task demands, and that there is an inverse relationship between the size of the attentional focus and the processing efficiency within that focus, due to limited attentional resources (Castiello \& Umiltà, 1990; Eriksen \& St. James, 1986; Eriksen \& Yeh, 1985). The larger the attentional focus, the lower the processing efficiency and the slower the detection of a stimulus subsequently appearing in the attended area. Furthermore, attention could be split and manipulated simultaneously into independent attentional foci on objects located in opposite hemifields (Castiello \& Umiltà, 1992). In addition, the location of the attentional focus is retinotopcially organized in the visual cortex (Brefczynski \& DeYoe, 1999; Somers, Dale, Seiffert, \& Tootell, 1999; Tootell, Hadjikhani, Hall, et al., 1998; Tootell, Hadjikhani, Mendola, Marrett, \& Dale, 1998). Finally, the extent of activated retinotopic visual cortex increased and the neural activity in a given subregion decreased as the size of the attentional focus increased (Müller, Bartelt, Donner, Villringer, \& Brandt, 2003).

In addition to the size of attentional focus, the detection of an object is influenced by the size of the object: the larger the object, the faster the detection time (Marzi, Mancini, Metitieri, \& Savazzi, 2006; Osaka, 1976; Ueno, 1978). Furthermore, it is the perceived, rather than the retinal, size that influences the detection of behavioral targets (Plewan, Weidner, \& Fink, 
2012; Sperandio, Savazzi, Gregory, \& Marzi, 2009; Sperandio, Savazzi, \& Marzi, 2010). For example, the Ponzo illusion refers to the phenomenon that when two lines of the same (retinal) size are placed over backgrounds with pictorial depth cues (e.g., a pair of converging lines), the seemingly farther line will be illusorily perceived as larger than the seemingly closer line. It has been suggested that in the Ponzo illusion, an object with a larger perceived size is detected more quickly and activates a larger area in the human primary visual cortex than does an object with smaller perceived size, although the two objects are of the same retinal size (Fang, Boyaci, Kersten, \& Murray, 2008; Murray, Boyaci, \& Kersten, 2006; Sperandio, Chouinard, \& Goodale, 2012; Sperandio et al., 2010).

A variety of optical size illusions are induced by pictorial depth cues, because a farther object would have to be larger than a closer one if both produce retinal images of the same size, according to the size-distance invariance law (Emmert, 1881; Holway \& Boring, 1941; Joynson, 1949; Ono, 1966). Therefore, the 2-D Ponzo illusion turns into a visual reality in 3-D space: Farther objects are both physically larger and perceived as larger than closer objects, although the farther and closer objects have the same retinal sizes. In the 3-D case, it is a reality, rather than an illusion, that a farther object is perceived as larger than a closer object of the same retinal size, or that a farther object is perceived as the same size as a closer one when the retinal size of the former is smaller than the retinal size of the latter. Size constancy makes a significant contribution to the perceived stability of the 3-D world: That is, the perceived size of an object remains relatively unchanged, in spite of changes in the retinal size of the object as it moves toward or away from the observer (Andrews, 1964; Gregory, 1997; Gregory \& Zangwill, 1987; Morgan, 1992; Slater, Mattock, \& Brown, 1990).

It remains unknown, however, whether and how the retinal size of a previous cue influences the process of attentional orienting toward a subsequent target in 2-D or 3-D space. To investigate this question, in the present study we varied the retinal size while matching the perceived size of two line segments/bars either under the 2-D Ponzo illusion or in 3-D reality, and adopted these stimuli as the cue display in the dot probe paradigm (e.g., Koster, Crombez, Verschuere, \& De Houwer, 2004; Mathews \& MacLeod, 2002; Watson \& Humphreys, 2000). The sizes of the line segments in the cue display of the dot probe paradigm determine the size of the attentional focus. That is, the two line segments in the cue display can unevenly capture attention, and the sizes of the line segments in the cue display can thus determine the size of the spatial area attended (Castiello \& Umiltà, 1992). Specifically speaking, in the 2-D condition, two parallel line segments under the Ponzo illusion were presented as the cue display in the dot probe paradigm, and the perceived sizes of the two line segments were matched by means of a pretest psychophysical experiment. When the perceived sizes of the two lines were matched in terms of psychophysical measures of the size of illusion, the retinal size of the seemingly farther line was smaller than that of the seemingly closer line. After the disappearance of the cue display, a red dot, which served as the target, could appear at the location of either of the line segments: the one with a larger or the one with a smaller retinal size (Fig. 1a). Similarly, in the cue display of the 3-D reality condition, the perceived (and physical) sizes were matched between farther and closer bars. When the perceived (and physical) sizes were matched, the retinal size of the farther bar was smaller than that of the closer bar, and the target could appear at the location of either the farther or the closer bar. Most critically, the retinal images were strictly matched between the 2-D illusion and 3-D reality conditions.

We predicted that if the retinal, rather than the perceived, size of the cue is what influences attentional orienting, a cue validity effect should be observed when the larger-retinal-size condition was contrasted with the smaller-retinal-size condition. With regard to the direction of the cue validity effect, on the basis of the zoom lens model, we predicted that if a cue with a larger retinal size cues a larger spatial area to be attended, the processing efficiency within the larger attentional focus should decrease, and accordingly, detecting targets appearing at the location of the cue with the larger retinal size should be slower than detecting targets at the location of the cue with the smaller retinal size. Otherwise, if the detection times were comparable for targets appearing at the locations of cues with larger and smaller retinal sizes, the perceived, rather than the retinal, size of the cue would be what influenced the attentional-orienting process. Moreover, although shifting between the 2-D Ponzo illusion and 3-D reality provided us with an opportunity to vary the retinal sizes while simultaneously matching the perceived sizes of the cues, the seemingly (or actually) closer line in the 2D (or 3-D) condition would always occupy a larger retinal area than the seemingly or actually farther line. Therefore, one might argue that the depth information, rather than the retinal size, of the cue display was what affected the attentionalorienting process. To rule out this possibility, in Experiment 2 we removed the 3-D background and placed both cues at either the farther or the closer position. We predicted that if similar patterns of results were observed in both the farther and the closer depth planes in Experiment 2, then retinal size per se, rather than depth information, would be what affected the attentional-orienting process. In addition, to further confirm the effect of retinal size on attentional orienting, we performed another 2-D control experiment (Exp. 3), in which both the pictorial depth background and the depth dimension were removed. We predicted that if the speed of attentional orienting still changed as a function of the size of the cue on a 2-D screen with a blank background, we could further confirm that the retinal size of the cue was what influenced the process of attentional orienting. 

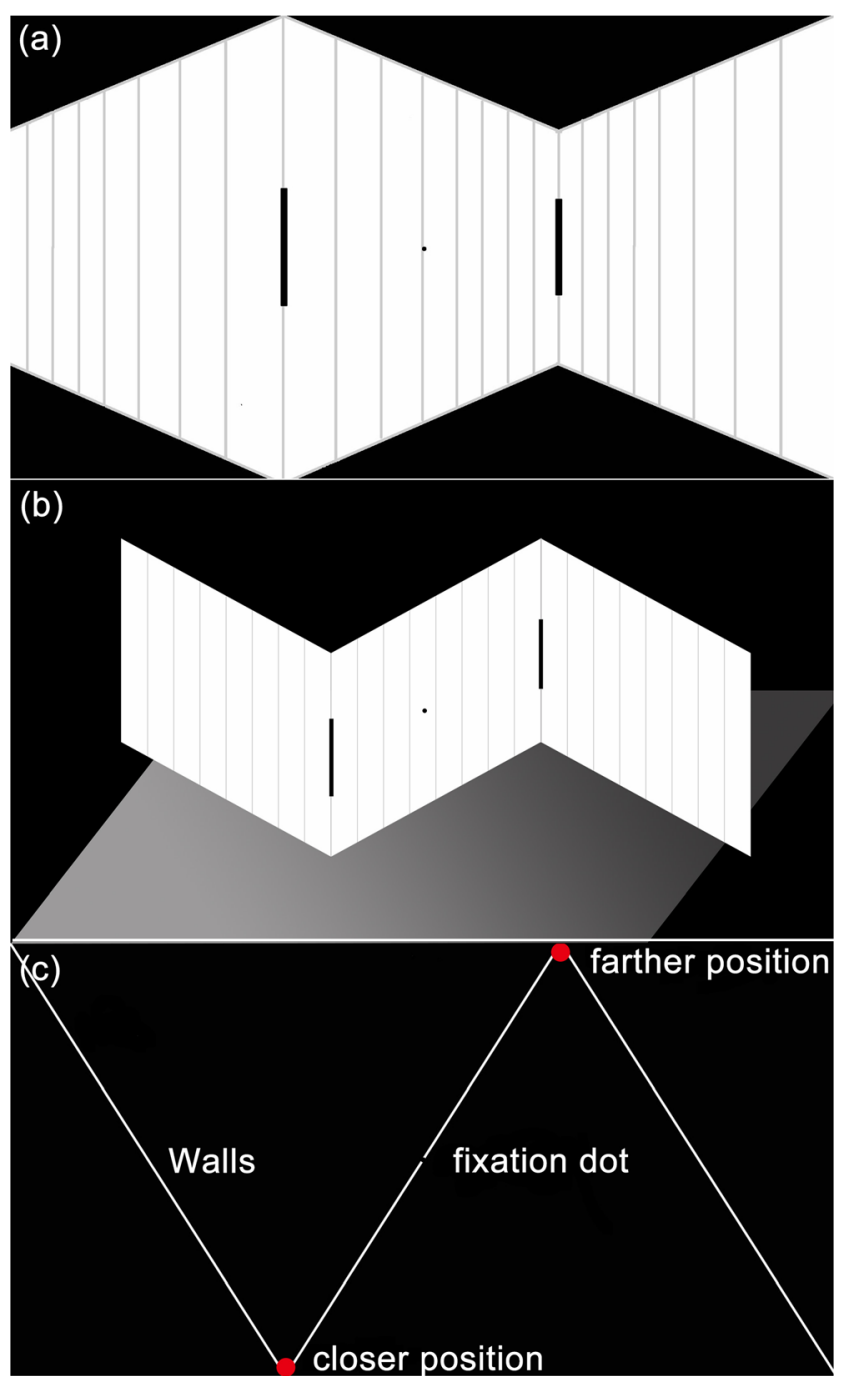

Fig. 1 a Frontal (retinal) view of the cue display in the 2-D and 3-D condition. The closer line is on the left side of the display. b Illustrative view of the cue display in the 2-D and 3-D conditions. The closer line is again on the left side of the display. $\mathbf{c}$ Top view of the target display in the 3-D condition

\section{Experiment 1: Retinal sizes varied (perceived sizes matched)}

In Experiment 1, we aimed to investigate the potential effect of the retinal size of the cue on attentional orienting in 2-D and 3-D space. The present experiment consisted of two parts: one psychophysical pretest and the main experiment. The psychophysical pretest was included to measure the size of the 2-D Ponzo illusion individually. For the 2-D illusion condition, by decreasing the length of the seemingly farther line or increasing the length of the seemingly closer line according to the derived size of illusion from the psychophysical pretest, we were able to match the perceived sizes of the two lines in the 2-D condition of the main experiment for each participant. For the 3-D condition, we simply put the same objects at farther and closer positions, so that the two objects were perceived as the same because of size constancy (Emmert, 1881; Holway \& Boring, 1941; Joynson, 1949; Ono, 1966).

\section{Method}

The psychophysical experiment: Measuring the size of 2-D Ponzo illusion individually

Participants Thirty volunteers (eight males, 22 females; $21 \pm$ 2 years old) participated in the present experiment. The participants were all right-handed and had normal or correctedto-normal visual acuity. None of them had a history of neurological or psychiatric disorders, and all participants gave informed consent prior to the experiment and were paid for their participation afterward.

Apparatus, stimuli, and procedure An ASUS LCD monitor was used in the experiment to present the experimental stimuli, with a resolution of 1,920 (horizontal) $\times 1,080$ (vertical) pixels at a $120-\mathrm{Hz}$ refresh rate. The size of the monitor was 27 in.

The 2-D stimuli used in the psychophysical pretest were adapted from the 3-D stimuli presented in the following main experiment. The virtual 3-D cue display included background walls, one closer and one farther black bar/line segment, and one central fixation ball at equal spatial distances from the two bars (Figs. 1a, b, and 2a). The 3-D objects were presented on a black background by custom-made Presentation scripts (Presentation Software package; Neurobehavioral Systems Inc., Albany, CA). For the 2-D display, the depth dimension was removed. Critically, the retinal images of the 2-D and 3-D displays were exactly the same (Figs. 1a and 2a). The background walls and the black fixation dot $\left(0.11^{\circ}\right.$ of visual angle in diameter) were presented throughout the experiment as the default display, and participants were instructed to fixate the central fixation without moving their eyes, at an approximate distance of $80 \mathrm{~cm}$ from the computer screen. The two parallel bars/line segments $\left(0.3^{\circ}\right.$ in width $)$ were $7.9^{\circ}$ of visual angle from the fixation dot (Figs. 1a, $\mathrm{b}$, and $2 \mathrm{a}$ ), and their initial lengths were randomly selected between $3.92^{\circ}\left[4.9^{\circ} *(1-.20)\right]$ and $5.88^{\circ}\left[4.9^{\circ} *(1+\right.$ $.20)]$ of visual angle ( $4.9^{\circ}$ of visual angle was the length of the line segment in the main experiment). In each trial, the length of one of the two lines was fixed, and the length of the other line was adjusted by the participants until they subjectively perceived the two lines as having the same size. The participants performed 128 trials in total; in 64 of the trials the seemingly closer line was in the left hemifield (Fig. 1a), and in the other 64 trials it was in the right hemifield (Fig. 1b). The size of the illusion for each participant was defined according to Formula 1: 


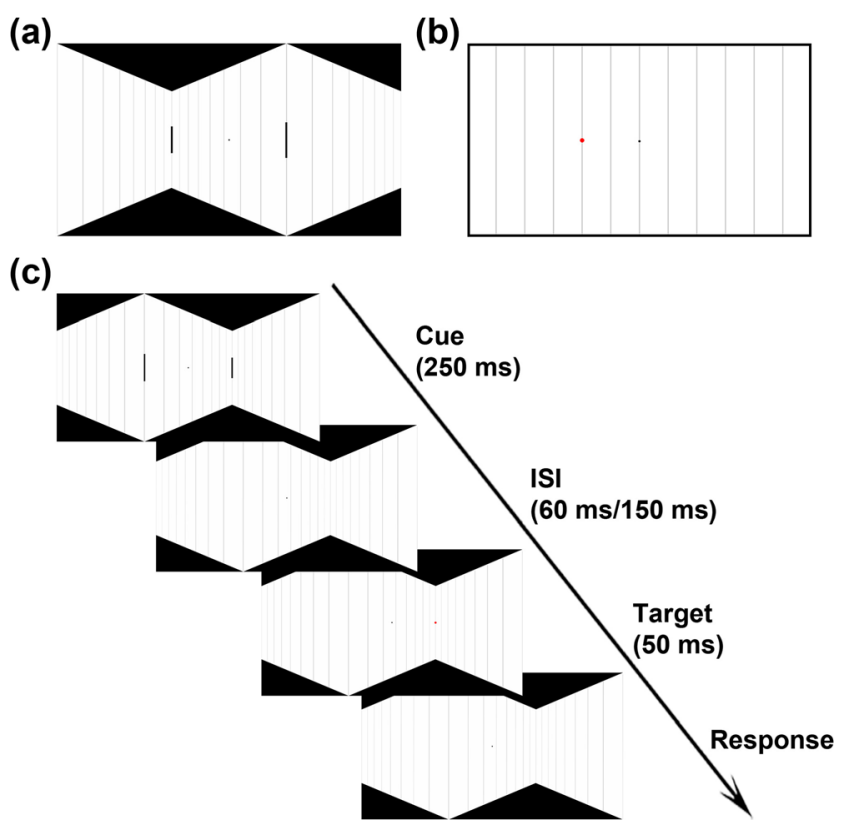

Fig. 2 a Frontal (retinal) view of the cue display in the 2-D and 3-D conditions. The closer line is on the right side of the display. b Frontal (retinal) view of the target display in Experiment 3. c Example of the timing and procedure of an experimental trial

$$
\begin{aligned}
& \text { Size of illusion }(i) \\
& \quad=\operatorname{Average}\left(\mathrm{L}_{\mathrm{sf} \_1} / \mathrm{L}_{\mathrm{sn} \_1}+\ldots+\mathrm{L}_{\mathrm{sf} \_n} / \mathrm{L}_{\mathrm{sn} \_n}+\ldots+\mathrm{L}_{\mathrm{sf}_{-} 64} / \mathrm{L}_{\mathrm{sn} \_64}\right),
\end{aligned}
$$

where Size of illusion $(i)$ is the size of the illusion for participant $i, \mathrm{~L}_{\mathrm{sf} n}$ is the physical length of the seemingly farther line segment after the participant's adjustment on trial $n$, and $\mathrm{L}_{\mathrm{sn} n}$ is the physical length of the seemingly closer line segment after the participant's adjustment on trial $n$. In each trial, the value of $\mathrm{L}_{\mathrm{sf} n}$ divided by $\mathrm{L}_{\mathrm{sn} n}$ was calculated. The size of the illusion was defined as the average value over 64 trials.

\section{The main experiment}

Participants The same group of participants as in the psychophysical experiment participated in the main experiment.

Apparatus, stimuli, and procedure A pair of 3-D glasses (NVIDIA 3D Vision 2), a 3-D Vision USB IR emitter, and an ASUS LCD monitor were used in the experiment to present virtual 3-D objects by delivering slightly disparate images to each eye (i.e., binocular disparity) when the participants were wearing the 3-D glasses. The ASUS LCD monitor had a resolution of 1,920 (horizontal) $\times 1,080$ (vertical) pixels at a 120 $\mathrm{Hz}$ refresh rate. The size of monitor was 27 in.

For the 3-D condition, the virtual 3-D cue display in each trial included background walls, one closer and one farther black bar, and one central fixation ball at equal spatial distances from the two bars (Figs. 1a, b, and 2a). The 3-D objects were presented on a black background using custommade Presentation scripts. For the 2-D condition, the depth dimension was removed. In the 2-D illusion condition, we matched the perceived sizes of the two lines for each participant in the following two ways: We either kept the length of the closer line at $4.9^{\circ}$ of visual angle and calculated the length of the farther line according to Formula 2 below, or we kept the length of the farther line at $4.9^{\circ}$ of visual angle and calculated the length of the closer line segment according to Formula 3. In the 3-D condition, according to the size-distance invariance law, the perceived size of an object was unchanged whether it was placed in far or near space. Therefore, we either moved the bar that was $4.9^{\circ}$ of visual angle in the far position to the near position, resulting in the retinal length of the closer bar being $12.98^{\circ}$, or moved the bar that was $4.9^{\circ}$ of visual angle in the near position to the far position, resulting in the retinal length of the farther bar being $1.85^{\circ}$. Therefore, although the retinal size of the seemingly (2-D) or the really (3-D) farther line was smaller than the retinal size of the seemingly or really closer line, their perceived sizes were matched.

Length of the farther line segment

$$
=4.9^{\circ} * \operatorname{Size} \text { of illusion }(i)
$$

Length of the closer line segment

$$
=4.9^{\circ} / \text { Size of illusion }(i)
$$

The background walls and the black fixation dot $\left(0.11^{\circ}\right.$ of visual angle in diameter) were presented throughout the experiment as the default display, and participants were instructed to fixate the central fixation without moving their eyes. The two parallel bars/line segments $\left(0.3^{\circ}\right.$ in width $)$ in the cue display were located $7.9^{\circ}$ of visual angle from the fixation dot (Figs. 1a and 2 a). A red dot $\left(0.29^{\circ}\right.$ in diameter) served as the target. For half of the trials, the closer end of the walls, and accordingly the closer bars/line segment, was presented in the left hemispace (3-D)/hemifield (2-D) (Fig. 1a), and vice versa for the other half of trials (Fig. 2a).

At the start of each trial, the cue display was presented for $250 \mathrm{~ms}$ (Fig. 2c). After an interstimulus interval (ISI) of either 60 or $150 \mathrm{~ms}$, which was employed to prevent participants from being able to predict the timing of the target's appearance after the cues' appearance, the target (the red dot) appeared at the spatial location of either the left or the right bar/line segment for $50 \mathrm{~ms}$. Participants were instructed to press a response button with the index finger of their right hand as quickly and accurately as possible when they detected the appearance of the target.

Experimental design A 2 (Dimension: 2-D vs. 3-D) $\times 2$ (Retinal Size: larger vs. smaller) $\times 2$ (ISI: 60 vs. $150 \mathrm{~ms}$ ) factorial design was adopted in the main experiment. We 
presented eight experimental conditions in total, with 24 trials in each of the conditions, and another 24 catch trials, in which no target was presented after the cue, were included. The catch trials were adopted to prevent participants from making false alarm responses during the time interval between the cue and the actual appearance of the targets. All of the trials were randomly mixed together.

\section{Results and discussion}

Incorrect responses and those with reaction times (RTs) exceeding three standard deviations above or below the mean $\mathrm{RT}$ in each condition were excluded from further analysis. The data of two participants were discarded because of their high false alarm rates on the catch trials (both higher than $10 \%$ ).

The RTs were submitted to a 2 (Dimension: $2-\mathrm{D}$ vs. $3-\mathrm{D}$ ) $\times$ 2 (Retinal Size: larger vs. smaller) $\times 2$ (ISI: $60 \mathrm{~ms}$ vs. $150 \mathrm{~ms}$ ) repeated measures ANOVA (Fig. 3a). The main effect of retinal size was significant, $F(1,27)=10.135, p=.004, \eta^{2}=$ .273 , indicating that responses to the subsequent targets at the location of the line with the larger retinal size $(302 \mathrm{~ms})$ were significantly slower than those to the targets at the location of the line with smaller retinal size $(294 \mathrm{~ms})$. The main effect of dimension was also significant, $F(1,27)=12.693, p=.001, \eta^{2}$ $=.32$, indicating that responses were significantly faster in the 2-D (290 ms) than in the 3-D (307 ms) condition, which might have resulted from the additional attentional shifts in depth in the 3-D condition, as compared to the 2-D condition (e.g., Kasai, Morotomi, Katayama, \& Kumada, 2003; Maringelli, McCarthy, Steed, Slater, \& Umiltà, 2001). The main effect of ISI was significant, $F(1,27)=30.918, p<.001, \eta^{2}=$ .534 , indicating that responses at the shorter ISI $(307 \mathrm{~ms})$ were significantly slower than responses at the longer ISI (290 ms). The interaction between dimension and ISI was significant, $F(1,27)=12.118, p=.002, \eta^{2}=.310$. Further tests on the simple effects showed that the RT difference between the shorter and longer ISIs was larger in the 3-D $(23 \mathrm{~ms})$ than in the 2 -D $(10 \mathrm{~ms})$ condition, $t(27)=3.481, p=.002$. The interaction between ISI and retinal size was marginally significant, $F(1,27)=4.171, p=.051, \eta^{2}=.134$. Further tests on the simple effects showed that at the longer ISI, there was a trend for responses to the targets at the location of the line with the larger retinal size $(293 \mathrm{~ms})$ to be slower than responses to the targets at the location of the line with the smaller retinal size (288 ms), $t(27)=1.863, p=.073, \eta^{2}=.114$. At the shorter ISI, the responses to the targets at the location of the line with the larger retinal size $(311 \mathrm{~ms})$ were significantly slower than the responses to the targets at the location of the line with the smaller retinal size $(301 \mathrm{~ms}), t(27)=3.555, p=.001, \eta^{2}=$ .312. Moreover, the RT difference induced by the different retinal sizes was marginally significantly larger at the shorter ISI $(10 \mathrm{~ms})$ than at the longer ISI $(5 \mathrm{~ms})$, $t(27)=2.042, p=.051$.
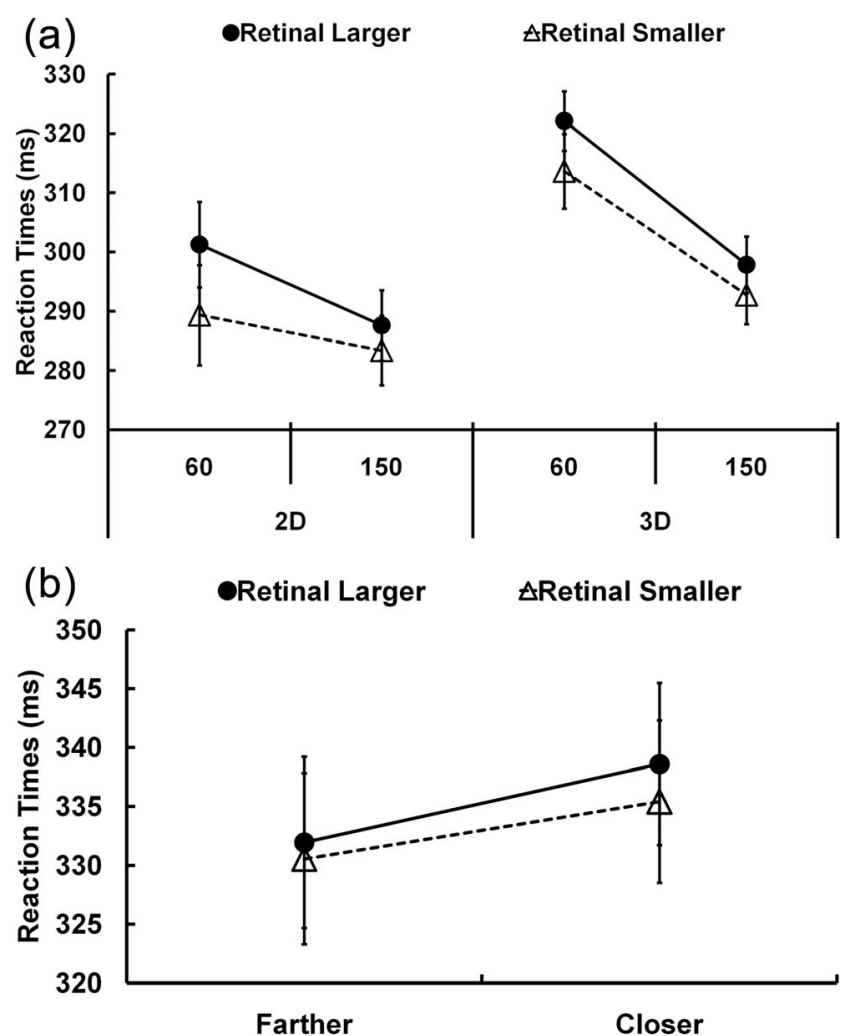

(c)

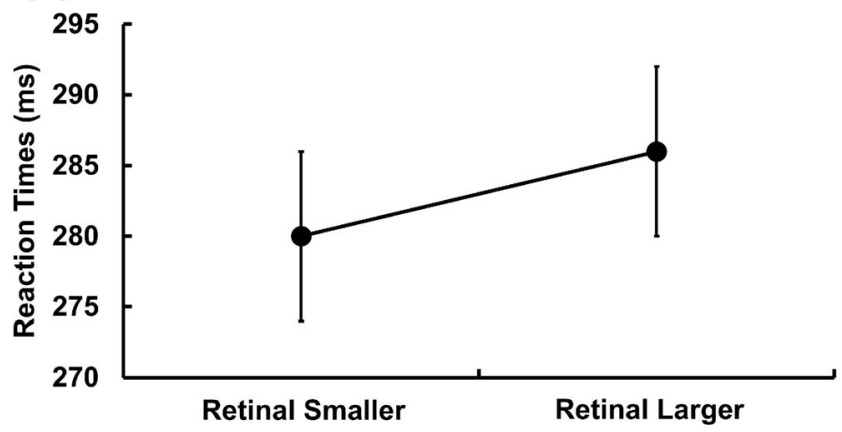

Fig. 3 a Mean reaction times (RTs) in Experiment 1. b Mean RTs in Experiment 2. c Mean RTs in Experiment 3. The error bars indicate the standard errors in each experimental condition

No other effects reached significance. These results suggested that the effect of retinal size on attentional orienting gradually decreased with increasing ISI.

\section{Experiment 2}

The results of Experiment 1 showed that the cue with a smaller retinal size facilitated the process of attentional orienting toward the subsequent target, relative to the cue with a larger retinal size. To further examine whether the effect above was caused by retinal size per se or by the depth information, we removed the background information and placed the cue display at either the farther or the closer depth plane in 
Experiment 2. Except for these differences, all other aspects of the paradigm and the stimuli were exactly the same as in Experiment 1. If the same pattern of results were obtained in both the farther and closer conditions, the influence of depth information could be ruled out.

\section{Method}

Participants Thirty volunteers (10 males, 20 females; $20 \pm$ 3 years old) participated in the present experiment. They were all right-handed and had normal or corrected-to-normal visual acuity. None of them had a history of neurological or psychiatric disorders, and all gave informed consent prior to the experiment and were paid for their participation afterward.

Apparatus, stimuli, and procedure The present experiment differed from Experiment 1 in two respects: (1) the background walls were removed and (2) the two line segments in the cue display were placed in either the farther or the closer depth plane at the same time (Fig. 4a and b). The rest of the apparatus and the stimuli were exactly the same as in Experiment 1.

Experimental design A 2 (Depth: farther vs. closer) $\times 2$ (Retinal Size: larger vs. smaller) factorial design was adopted in the present experiment. We presented 56 trials in each of the four experimental conditions, and another 28 catch trials, in which no target was presented after the cue. The catch trials were adopted to prevent participants from making false alarm responses during the time interval between the cue and the actual appearance of the targets. The far and close blocks were separate, and the order of the two blocks was counterbalanced between participants.

\section{Results and discussion}

Incorrect responses and RTs exceeding three $S D$ s above or below the mean RT in each condition were excluded from further analysis. The data of two participants were discarded because of their high false alarm rates on the catch trials (both higher than $10 \%$ ).

The RTs were submitted to a 2 (Depth: farther vs. closer) $\times$ 2 (Retinal Size: larger vs. smaller) repeated measures ANOVA (Fig. 3b). The main effect of size was significant, $F(1,27)=$ $5.752, p=.024, \eta^{2}=.176$, indicating that RTs to the targets at the location of the line with the larger retinal size $(335 \mathrm{~ms})$ were significantly slower than RTs to the targets at the location of the line with the smaller retinal size $(333 \mathrm{~ms})$. The main effect of depth was not significant, $F(1,27)=1.359, p=.254$. No other effects reached significance. (a)

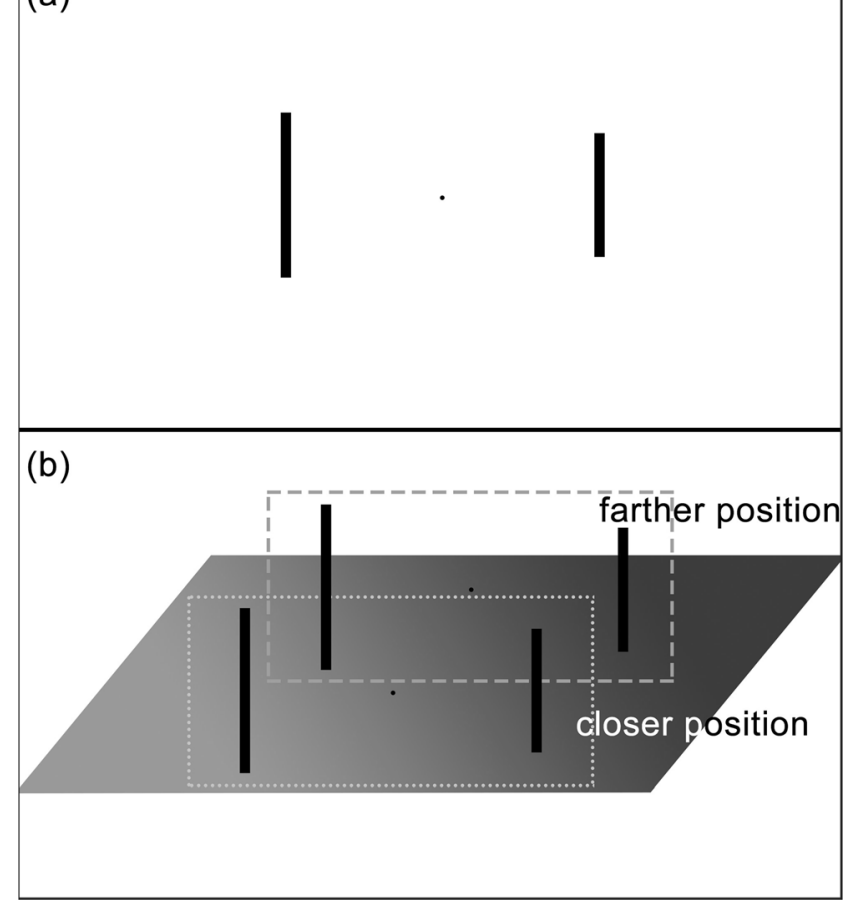

Fig. 4 a Frontal (retinal) view of the cue display in Experiment 2. b Illustration of the cue displays in the farther and closer positions

\section{Experiment 3}

The results in Experiments 1 and 2 showed that objects with a smaller retinal size more significantly facilitated the process of attentional orienting to the subsequent target at its location than did objects with a larger retinal size. To further test this, we ran a 2-D control experiment (Fig. 2b) in which the depth cue (i.e., the background walls) was removed from the 2-D Ponzo illusion. Except for this difference, all other aspects of the stimuli, including the lengths of the two lines, were exactly the same as in the 2-D condition of Experiment 1. Therefore, after the depth cue was removed, the retinal sizes of the two lines directly determined the perceived size - that is, the larger the retinal size, the larger the perceived size. We predicted that if objects with a smaller retinal size more significantly facilitate the process of attentional orienting to the subsequent target at its location than do objects with a larger retinal size, we should replicate the previous pattern of results, of facilitation for the line with smaller retinal (and perceived) size, in the present 2-D control experiment.

\section{Method}

Participants The same group of participants as in Experiment 1 took part in the present experiment.

Apparatus, stimuli, and procedure The present experiment differed from Experiment 1 in two respects: (1) only the 2-D 
condition was included in the present experiment, and (2) the depth cue - that is, the background wall — was removed from this 2-D condition.

Experimental design A one-factorial design with two levels (Size of the Cue: larger vs. smaller) was adopted in the present experiment.

\section{Results and discussion}

Incorrect responses and RTs exceeded three SDs above or below the mean RT in each condition were excluded from further analysis. The data of two participants were discarded because of their high false alarm rates on the catch trials (both higher than $10 \%$ ).

A paired-sample $t$ test (larger vs. smaller) was carried out on the RT data (Fig. 3c). A significant difference was revealed, $t(27)=4.087, p<.001$, indicating that responses to the subsequent targets at the location of the line with the larger size (286 ms) were significantly slower than responses to the targets at the location of the line with the smaller size $(280 \mathrm{~ms})$. The present results thus replicated and confirmed the results in Experiment 1.

\section{General discussion}

By combining the dot probe task with either the 2-D Ponzo illusion induced by pictorial depth cues or a virtual 3-D world in which the 2-D Ponzo illusion turned into visual reality, we tested the potential effect of the retinal versus perceived size of the cue on attentional orienting. The results in Experiment 1 showed that with the perceived size of the cue being kept constant, the larger the retinal size of the cue, the slower the responses to the subsequent targets at the cued location, and that this cue validity effect existed in both 2-D and 3-D space with comparable sizes (Fig. 3a). Furthermore, since in both the 2-D Ponzo illusion condition and the 3-D condition of Experiment 1, with the perceived sizes of the two lines being kept constant, the line with a larger retinal size was always the closer line, it could be that attentional orienting toward closer targets was significantly facilitated, relative to attentional orienting toward the farther targets. To rule out this possibility, in Experiment 2, we removed the background 3-D wall and placed the two line segments in the cue display together in either the farther or the closer depth plane. The results of Experiment 2 showed a pattern consistent with those of Experiment 1 -that is, attentional orienting to the position of the cue with the smaller size was significantly facilitated, as compared to orienting to the position of the cue with a bigger size in both the farther and closer depth planes, and there was no significant difference between the farther and closer conditions (Fig. 3b). Therefore, the retinal size per se, rather than the depth information, of the cue was what facilitated attentional orienting to the spatial locations cued with smaller as compared to larger cues. The results of the 2-D control experiment (Exp. 3) further confirmed that objects with a smaller retinal size more significantly facilitated the process of attentional orienting to the subsequent target at its location than did objects with a larger retinal size (Fig. 3c).

In one of the previous studies using a simple detection task, two boxes of different sizes were used as the cues, and participants were instructed to detect the appearance of a target that could appear in either of the two boxes with equal probabilities (Castiello \& Umiltà, 1992). The results showed that responses to the target appearing in the bigger box were significantly slower than responses to the target in the smaller box, which is consistent with our results in Experiments 1, 2, and 3. However, since all of the previous relevant studies had adopted 2-D displays, in which larger retinal sizes led to larger perceived sizes, it remained unclear whether the retinal or the perceived size of the cue was what caused the cue validity effect during attentional orienting. Therefore, together with previous evidence, our results in Experiments 1, 2, and 3 suggest that the retinal size of the cue plays a critical role in guiding attentional orienting. According to the zoom lens model of visuospatial attention, the size of the attentional focus is inversely correlated with the processing efficiency of the attention system (Castiello \& Umiltà, 1990; Eriksen \& St. James, 1986; Eriksen \& Yeh, 1985): The larger the spatial area attended, the lower the processing efficiency. In the present study, the retinal size of the cue might have determined the size of the attentional focus. The cue with a larger retinal size might have led participants to attend to a larger spatial area, which accordingly reduced the visual processing efficiency within the attentional focus and slowed down the responses to the targets at the location of the cues with a larger retinal size.

In addition to the size of the attentional focus, the processing efficiency of the attention system is also influenced by the saliency (e.g., as indexed by the size) of the object (Henderson \& Hollingworth, 1999; Theeuwes, 2010). It has been well documented that the perceived size, rather than the retinal size, of a target influences the detection time: With the retinal size being kept constant, the larger the perceived size, the faster the detection time (Plewan et al., 2012; Sperandio et al., 2009; Sperandio et al., 2010). In the present study, to rule out the effect of the size of the target on RTs, the retinal size of the target was set as small as possible $\left(0.29^{\circ}\right.$ in diameter $)$, and participants were instructed to detect the appearance of the target on the basis of its salient red color.

Taken together, to investigate whether the retinal or perceived size of a cue influences attentional orienting in 2-D and 3-D space, we combined the dot probe paradigm with either the 2-D Ponzo illusion or 3-D reality. By varying the retinal size of a stimulus while keeping the perceived size 
constant, we found that the retinal, rather than the perceived, size of the cue plays an important role in attentional orienting: The larger the retinal size of the cue, the slower the attentional orienting to targets at the location of that cue.

Author note This work was supported by grants from the Natural Science Foundation of China (No. 31371127) and the National Natural Science Foundation of China (Nos. J1210024 and J1310031). Q.C. is supported by the Program for New Century Excellent Talents at the University of China (Grant No. NCET-12-0645) and by the Guangdong Province Universities and Colleges Pearl River Scholar Funded Scheme (2014).

\section{References}

Andrews, D. (1964). Error-correcting perceptual mechanisms. Quarterly Journal of Experimental Psychology, 16, 104-115.

Brefczynski, J. A., \& DeYoe, E. A. (1999). A physiological correlate of the "spotlight" of visual attention. Nature Neuroscience, 2, 370-374. doi: $10.1038 / 7280$

Castiello, U., \& Umiltà, C. (1990). Size of the attentional focus and efficiency of processing. Acta Psychologica, 73, 195-209.

Castiello, U., \& Umiltà, C. (1992). Splitting focal attention. Journal of Experimental Psychology: Human Perception and Performance, 18, 837-848. doi:10.1037/0096-1523.18.3.837

Emmert, E. (1881). Grossenverhältnisse der Nachbilder. Klinische Monatsblatter für Augen-heilkunde, 19, 443-450.

Eriksen, C. W., \& St. James, J. D. (1986). Visual attention within and around the field of focal attention: A zoom lens model. Perception \& Psychophysics, 40, 225-240. doi:10.3758/BF03211502

Eriksen, C. W., \& Yeh, Y.-Y. (1985). Allocation of attention in the visual field. Journal of Experimental Psychology: Human Perception and Performance, 11, 583-597. doi:10.1037/0096-1523.11.5.583

Fang, F., Boyaci, H., Kersten, D., \& Murray, S. O. (2008). Attentiondependent representation of a size illusion in human V1. Current Biology, 18, 1707-1712. doi:10.1016/j.cub.2008.09.025

Gregory, R. L. (1997). Eye and brain: The psychology of seeing. Princeton: Princeton University Press.

Gregory, R. L., \& Zangwill, O. L. (1987). The Oxford companion to the mind. Oxford: Oxford University Press.

Henderson, J. M., \& Hollingworth, A. (1999). High-level scene perception. Annual Review of Psychology, 50, 243-271. doi:10.1146/ annurev.psych.50.1.243

Holway, A. H., \& Boring, E. G. (1941). Determinants of apparent visual size with distance variant. American Journal of Psychology, 54, 21-37.

Joynson, R. (1949). The problem of size and distance. Quarterly Journal of Experimental Psychology, 1, 119-135.

Kasai, T., Morotomi, T., Katayama, J., \& Kumada, T. (2003). Attending to a location in three-dimensional space modulates early ERPs. Cognitive Brain Research, 17, 273-285.

Koster, E. H., Crombez, G., Verschuere, B., \& De Houwer, J. (2004). Selective attention to threat in the dot probe paradigm: Differentiating vigilance and difficulty to disengage. Behavior Research and Therapy, 42, 1183-1192.
Maringelli, F., McCarthy, J., Steed, A., Slater, M., \& Umiltà, C. (2001). Shifting visuo-spatial attention in a virtual three-dimensional space. Cognitive Brain Research, 10, 317-322.

Marzi, C. A., Mancini, F., Metitieri, T., \& Savazzi, S. (2006). Retinal eccentricity effects on reaction time to imagined stimuli. Neuropsychologia, 44, 1489-1495. doi:10.1016/j. neuropsychologia.2005.11.012

Mathews, A., \& MacLeod, C. (2002). Induced processing biases have causal effects on anxiety. Cognition and Emotion, 16, 331-354.

Morgan, M. J. (1992). On the scaling of size judgements by orientational cues. Vision Research, 32, 1433-1445.

Müller, N. G., Bartelt, O. A., Donner, T. H., Villringer, A., \& Brandt, S. A. (2003). A physiological correlate of the "Zoom Lens" of visual attention. Journal of Neuroscience, 23, 3561-3565.

Murray, S. O., Boyaci, H., \& Kersten, D. (2006). The representation of perceived angular size in human primary visual cortex. Nature Neuroscience, 9, 429-434. doi:10.1038/nn1641

Ono, H. (1966). Distal and proximal size under reduced and non-reduced viewing conditions. American Journal of Psychology, 79, 234-241.

Osaka, N. (1976). Reaction time as a function of peripheral retinal locus around fovea: Effect of stimulus size. Perceptual and Motor Skills, 42, 603-606

Plewan, T., Weidner, R., \& Fink, G. R. (2012). The influence of stimulus duration on visual illusions and simple reaction time. Experimental Brain Research, 223, 367-375. doi:10.1007/s00221-012-3265-7

Slater, A., Mattock, A., \& Brown, E. (1990). Size constancy at birth: Newborn infants' responses to retinal and real size. Journal of Experimental Child Psychology, 49, 314-322.

Somers, D. C., Dale, A. M., Seiffert, A. E., \& Tootell, R. B. (1999) Functional MRI reveals spatially specific attentional modulation in human primary visual cortex. Proceedings of the National Academy of Sciences, 96, 1663-1668.

Sperandio, I., Chouinard, P. A., \& Goodale, M. A. (2012). Retinotopic activity in $\mathrm{V} 1$ reflects the perceived and not the retinal size of an afterimage. Nature Neuroscience, 15, 540-542. doi:10.1038/nn. 3069

Sperandio, I., Savazzi, S., Gregory, R. L., \& Marzi, C. A. (2009). Visual reaction time and size constancy. Perception, 38, 1601-1609.

Sperandio, I., Savazzi, S., \& Marzi, C. A. (2010). Is simple reaction time affected by visual illusions? Experimental Brain Research, 201, 345-350. doi:10.1007/s00221-009-2023-y

Theeuwes, J. (2010). Top-down and bottom-up control of visual selection. Acta Psychologica, 135, 77-99. doi:10.1016/j.actpsy.2010.02. 006

Tootell, R. B. H., Hadjikhani, N., Hall, E. K., Marrett, S., Vanduffel, W., Vaughan, J. T., \& Dale, A. M. (1998). The retinotopy of visual spatial attention. Neuron, 21, 1409-1422. doi:10.1016/S08966273(00)80659-5

Tootell, R. B. H., Hadjikhani, N. K., Mendola, J. D., Marrett, S., \& Dale, A. M. (1998). From retinotopy to recognition: fMRI in human visual cortex. Trends in Cognitive Sciences, 2, 174-183.

Ueno, T. (1978). Temporal summation in human vision: Simple reaction time measurements. Perception \& Psychophysics, 23, 43-50.

Watson, D. G., \& Humphreys, G. W. (2000). Visual marking: Evidence for inhibition using a probe-dot detection paradigm. Perception \& Psychophysics, 62, 471-481. doi:10.3758/BF03212099 